\title{
Detección oportuna de cáncer cérvico-uterino
}

\section{Detection program for cervical-uterine cancer}

\section{Detecção precoce de câncer cérvice-uterino}

\author{
Edison Mauricio Herrera Conza ${ }^{1}$ \\ maurik-h7@hotmail.com \\ ORCID: 0000-0002-2978-1379
}

Lizette Espinosa Martín²
lespinosam@ucacue.edu.ec
ORCID: 0000-0002-3455-4437

\author{
Zoila Katherine Salazar Torres ${ }^{2}$ \\ zsalazart@ucacue.edu.ec \\ ORCID: 0000-0002-7663-8049
}

\author{
Karla Alexandra Aspiazu Hinostroza ${ }^{2}$ \\ kaspiazuh@ucacue.edu.ec \\ ORCID: 0000-0002-6016-4109
}

1Ministerio de Salud del Ecuador, Unidad de Medicina General, Ecuador

2Universidad Católica de Cuenca, Ecuador

Recibido 31 de agosto 2020 | Arbitrado y aceptado 29 de septiembre 2020 | Publicado 22 de diciembre 2020

\begin{abstract}
RESUMEN
Introducción: en los países subdesarrollados, los programas de tamizaje apoyados en la "citología" como el de "Detección Oportuna del Cáncer Cérvicouterino", no han tenido el éxito esperado, debido a que se solicitan múltiples recursos para ejecutar cada uno de los pasos del programa, y no siempre se cuenta con el personal y la infraestructura necesaria. Objetivo: determinar la frecuencia del cumplimiento del programa de Detección Oportuna del Cáncer cérvicouterino. Metodología: se realizó una revisión bibliográfica se incluyeron investigaciones que proporcionaron información cuantitativa sobre el Programa de Detección Oportuna del Cáncer Cérvicouterino de las unidades de salud del Ministerio Salud Pública. La información se recolectó de artículos originales de revistas indexadas, y que se encontraron en la base de datos científicas como Scopus, Scielo y Google académico. Se encontraron 116 artículos, pero solo 17 resultaron ser elegibles y válidos para el estudio. Resultados: el incumplimiento de la cobertura del programa DOCcu a nivel nacional fue del $41,5 \%$ para el test de Papanicolaou, y de inmunizaciones contra el VPH fue del $15,91 \%$ para las niñas de 9 años, y en mujeres de más edad fue del 60\%, esto hasta el año 2018, a partir de esas fechas no hay registros, ni tampoco de las otras pruebas. El grupo de edad que más participó en DOCcu son las mujeres de 45 hasta los 49 años, con instrucción superior. Conclusiones: el "Programa de Detección Oportuna de cáncer", en relación al Paptest y la vacunación del VPH en Ecuador aún es deficiente.
\end{abstract}

Palabras clave: Programas nacionales de salud; evaluación de programas y proyectos de salud; neoplasias del cuello uterino; prueba del Papanicolaou

\begin{abstract}
Introduction: in underdeveloped countries, screening programs supported by "cytology" such as the "Timely Detection of Cervical Cancer" have not had the expected success, because multiple resources are requested to execute each of the steps of the program, and the necessary staff and infrastructure are not always in place. Objective: to determine the frequency of compliance with the program for the Timely Detection of Cervical Cancer. Methodology: a bibliographic review was carried out, and investigations were included that provided quantitative information on the Program for the Timely Detection of Cervical Cancer of the health units of the Ministry of Public Health. The information was collected from original articles from indexed journals, which were found in scientific databases such as Scopus, Scielo and academic Google 116 articles were found, but only 17 were found to be eligible and valid for the study. Results: non-compliance with the DOCcu program coverage at the national level was $41.5 \%$ for the Papanicolaou test, and immunizations against HPV was $15.91 \%$ for 9 -year-old girls, and in older women It was $60 \%$, this until 2018 , as of those dates there are no records, nor of the other tests. The age group that most participated in DOCcu are women from 45 to 49 years old, with higher education. Conclusions: the "Program for the Timely Detection of cancer", in relation to the Paptest and HPV vaccination in Ecuador is still deficient.
\end{abstract}

Key words: National health programs; evaluation of health programs and projects; uterine cervical neoplasms; Pap Smear
EH: Médico Ministerio de Salud Pública. Unidad de Medicina General Bombero Voluntario del Benemerito Cuerpo de Bomberos Chaguarpamba. Ministerio de salud pública del Ecuador. Ecuador.

ZS: Magister en Investigación de la Salud, Especialista en Ginecología y Obstetricia Docente Universidad Católica de Cuenca. Universidad Católica de Cuenca. Ecuador.

LE: Especialista en Medicina Interna. Universidad Católica de Cuenca Coordinadora de Investigación de la Unidad Académica de salud y Bienestar. Universidad Católica de Cuenca. Ecuador.

KA: Master en Investigación Médica Clínica y Experimental. Universidad Católica de Cuenca. Ministerio de Salud Pública del Ecuador. Ecuador. 
EH: Médico Ministerio de Salud Pública. Unidad de Medicina General Bombero Voluntario del Benemerito Cuerpo de Bomberos Chaguarpamba. Ministerio de salud pública del Ecuador. Ecuador.

ZS: Magister en Investigación de la Salud, Especialista en Ginecología y Obstetricia Universidad Católica de Cuenca. Ecuador.

LE: Especialista en Medicina Interna Universidad Católica de Cuenca Coordinadora de Investigación de la Unidad Académica de salud y Bienestar. Universidad Católica de Cuenca. Ecuador.

KA: Master en Investigación Médica Clínica y Experimental. Universidad Católica de Cuenca. Ministerio de Salud Pública del Ecuador. Ecuador.

\section{RESUMO}

Introdução: em países subdesenvolvidos, programas de rastreamento apoiados por "citologia", como a "Detecção Oportuna de Câncer do Colo Uterino", não tiveram o sucesso esperado, pois vários recursos são solicitados para executar cada uma das etapas do programa, e o pessoal necessário e a infraestrutura nem sempre está instalada. Objetivo: determinar a frequência de cumprimento do programa de Detecção Oportunidade de Câncer do Colo Uterino. Metodologia: foi realizada uma revisão bibliográfica, incluindo-se investigações que fornecessem informações quantitativas sobre o Programa de Detecção Atempada do Câncer do Colo Uterino das unidades de saúde do Ministério da Saúde Pública. As informações foram coletadas a partir de artigos originais de periódicos indexados, encontrados em bases de dados científicas como Scopus, Scielo e Google acadêmico. 116 artigos foram encontrados, mas apenas 17 foram considerados elegíveis e válidos para o estudo. Resultados: o não cumprimento da cobertura do programa DOCcu em nível nacional foi de $41,5 \%$ para o teste de Papanicolau e a imunização contra o HPV foi de $15,91 \%$ para meninas de 9 anos, e em mulheres mais velhas foi de $60 \%$, até 2018 , a partir dessas datas não há registros, nem dos demais testes. A faixa etária que mais participa do DOCu são mulheres de 45 a 49 anos, com ensino superior. Conclusões: o "Programa de Detecção Oportuna de Câncer", em relação à vacinação Paptest e HPV no Equador, ainda é deficiente.

Palavras-chave: Programas nacionais de saúde; avaliação de programas e projetos de saúde; neoplasias cervicais; esfregaço de Papanicolau

\section{INTRODUCCIÓN}

A nivel mundial, en relación a los tipos de cáncer que afectan a la mujer, el cáncer cervical (CC) o de cuello uterino (CCU) ocupa el cuarto lugar, y se origina en las células que revisten el epitelio cervical (1). En año 2018, hubo 570.000 nuevos casos de CC, con una tasa de mortalidad del 7,5\%; registrando 311.000 fallecimientos, de estas el $85 \%$ en áreas pocos desarrolladas (2-5). Con la introducción del "programa de vacunación contra el virus del papiloma humano (VPH)", la tasa global se redujo de 10,2 a 8,5 casos por 100.000 mujeres (6), solo en los Estados Unidos se disminuyó un $50 \%$ en las últimas tres décadas, relacionando a la eficacia de las pruebas del Papanicolaou (1). Sin embargo, en naciones en vías de desarrollado, el CCU ocupa el primer lugar de los cánceres ginecológicos, tal es el caso que, en América Latina, el CCU es el segundo tipo cáncer que afecta a la población femenina, con 8,7 muertes por 100.000 mujeres, y el $75 \%$ se origina en países como Brasil,
México, Colombia, Perú, Venezuela y Argentina. Sin embargo, la mortalidad, es más alta en Guyana con 21,9, Bolivia con 21,0 y Nicaragua con 18,3 (7).

El CCU, por su magnitud $y$ trascendencia en las esferas individual, familiar, social y económica sigue siendo un problema en la "Salud Pública". Esta enfermedad es motivo de dolor físico y sufrimiento para las mujeres enfermas y sus familiares, y los costos del diagnóstico, tratamiento y control se sitúan entre los más altos en el contexto de la medicina, motivo por lo que se le estima de tipo catastrófico a nivel colectivo e institucional (8). Ecuador en el año 2012 el CCU se adjudicó el segundo lugar después del cáncer de mama, con una incidencia de 2094 casos y una mortalidad de 1026 mujeres (8). Para el año 2014 se le atribuyeron 650 defunciones a esta patología, siendo la edad media al momento del diagnóstico 54 años, siendo la población más afectada la que vivía en condiciones socioeconómicas deficitarias llegando hacer 10 veces mayor (9). 
El sistema de salud ecuatoriana, ha elaborado la Estrategia Nacional para la Atención Integral del Cáncer en Ecuador, con el fin de disminuir la morbimortalidad, y apoyar en la calidad de vida de los pacientes con cáncer. Es por ello, que las estrategias que se establecieron para la prevención CCU, parten con la realización del cribado con Papanicolaou cada 3 años en mujeres de 21 a 65 años, y en mujeres de 30 a 65 años; además, de ser posible, realizar el tamizaje con citología y pruebas moleculares para ADN de VPH cada 5 años $(10,11)$. Además, la atención primaria comprende la vacunación contra el VPH desde los 9 a 13 años, la educación sobre la sexualidad adaptada a la edad y la cultura, la promoción del uso y suministro de profilácticos a quienes tengan actividad sexual. La prevención secundaria parte en mujeres mayores a 30 años mediante la prueba del tamizaje y tratamiento, según sea necesario a través de la "Inspección Visual con Ácido Acético" (IVAA), de bajo costo, seguida de crioterapia (11).

En el contexto ecuatoriano, en la provincia del Tungurahua, se valoró la calidad de Programa de Detección Oportuna del Cáncer Cérvicouterino (DOCcu), en un universo de 1726 pacientes que acudieron a realizarse el Paptest (test de Papanicolaou). Se determinó que el $81,8 \%$ de las citología fueron realizadas por profesionales en el área de obstetricia y el $18,2 \%$ por médicos con postgrados en el ámbito de la medicina familiar, en este sentido se detectaron fallas de inconsistencias del $54,7 \%$ en el manejo de los resultados negativos de las pacientes, es entonces que se estableció que no se cuenta con un equipo multidisciplinario de profesionales, por lo que las pruebas presentan fallos en los procesos de captación de las mujeres en edad de riesgo, toma, obtención, envío de muestras y manejo de resultados (12).
Otro estudio, aborda los factores que influyen en la cobertura del DOCcu en mujeres de edad fértil de la ciudad de Cuenca; como resultado se evidenció que la cobertura del programa, fue del $64,4 \%$ (2.855), quedando el $35,6 \%$ sin realizar el Paptest. Se destacó también, entre los elementos que influyeron al no uso del servicio fue el miedo u obligación para acceder a otros programas, falta de tiempo, privacidad, maltrato del personal, resultados tardíos. Sin embargo, el nivel de satisfacción fue 76,4\% (13).

Tomando en consideración lo anterior mencionado, se puede extrapolar que, pese a las medidas implementadas por los entes regulatorios de la salud en cada uno de los países, existe factores que inciden negativamente en los DOCcu (14). Así lo afirma, un estudio de "Prevalencia de genotipos del VPH en mujeres de la provincia del Azuay", el cual señala que en el año 2014 se elevó la tasa en un 17,81\%, $\mathrm{y}$ fue notorio el incremento del número de pacientes con CCU; esto pudo ser originado por el bajo índice de efectividad de las medidas de detección y de prevención en los centros de salud (15). Así mismo, de acuerdo al estudio efectuado por GLOBOCAN, "el CCU sigue siendo la tercera causa de muerte por cáncer a nivel mundial en el sexo femenino entre los $35 \mathrm{y}$ 64 años" (16), y factor de riesgo es el VPH, con una prevalencia del $50 \%$ a $80 \%$ en mujeres con actividad sexual previa (17), ya que el $80 \%$ de las mujeres y hombres se han contagiado en algún momento de sus vidas, otros factores asociados están la edad temprana en la primera relación sexual, múltiples compañeros (as) sexuales pasados y presentes, inmunosupresión o infección por VIH, exposición a Herpes virus, Chlamydia, entre otras, además de inhalar humo de tabaco, leña u otro producto que contenga sustancias 
cancerígenas (2). El pico máximo para la adquisición del VPH tanto por hombres como por mujeres es al poco tiempo de volverse sexualmente activos, y rara vez provoca síntomas (18), se considera una patología multifactorial y de lenta progresión, que se desarrolla con su asociación a la infección persistente por algunos de los tipos del virus del VPH descritos como de alto riesgo, y en ausencia de tratamiento, aumenta la posibilidad de desarrollar cáncer (19).

Del 50 al $80 \%$ de pacientes contagiadas por el VPH se considera que sólo el 5\% desarrollaran una infección persistente, las cuales pueden generar lesión intraepitelial escamosa de bajo grado (LIEBG) o de alto grado (LIEAG) (20, 21). Es transcendental destacar que, en la mayoría de los casos, sobre todo en las mujeres jóvenes, las infecciones por VPH de alto riesgo oncogénico son transitorias $\mathrm{y}$ remiten solas sin producir ninguna displasia $(22,20)$. El VPH está además asociado a una variedad de lesiones malignas como el carcinoma invasor de la zona ano-genital: pene, ano, cuello uterino, vulva y los subtipos 16 y $18 \mathrm{VPH}$, los que han sido identificados como los más prevalentes, alcanzando esta relación hasta un 66\% (23).

La detección a través de las pruebas, está basada en 3 tipos que son recomendadas por la OMS como son las pruebas de VPH para tipos de VPH de alto riesgo, inspección visual con ácido acético (IVAA), prueba convencional (Pap) y citología basada en líquido (LBC), si las pruebas tuvieran una detección positiva, se emplea el tratamiento de lesiones precancerosas, mediante el uso de la crioterapia y procedimiento de escisión electro quirúrgica con asa (LEEP), en caso de lesiones avanzadas, los casos deben ser derivados para investigaciones adicionales y manejo adecuado (23-25).

En lo que corresponde a las pruebas de tamizaje se ha utilizado el Papanicolaou (muestra citológica exfoliativa cérvico vaginal), esta ha reducido la incidencia y mortalidad del CC en por lo menos $80 \%$, siendo este introducido hace ya 30 años en los países en desarrollo, y se recomienda realizarlo cada tres años en mujeres entre 21 y 29 años (26). La prueba test de detección VPH es un método que se utiliza para detectar el ADN viral del VPH en las células de cuello cervical, asimismo, se maneja como ensayo de seguimiento después del tratamiento de NIC II y NIC III (27).

Los tratamientos de dichas pruebas positivas conllevan a acciones de crioterapia, siendo este un procedimiento en el que no se requiere anestesia, se usa óxido nitroso o dióxido de carbono como refrigerante. Su eficacia en las tasas de remisión varía según el grado de lesión, evidenciándose que las displasias leves pueden remitir entre un $90,9-100 \%$, las displasias moderadas un $75,0-95,9 \%$ y las displasias severas, 71,0 - 91,7\% (28). La conización es una forma extensa de una biopsia de cuello uterino, en este procedimiento se extrae una cuña de tejido con forma de cono del cuello uterino y se examina bajo un microscopio, tomando una muestra endocervical para evaluar el resto del canal endocervical (27).

Otros tratamientos como el láser de bióxido de carbono, se utiliza un rayo láser para destruir el tejido del área de transformación, en donde se la destrucción del tejido con láser puede controlarse por la duración de la exposición, el éxito de este método es de un $95 \%$ al $96 \%$ veraz (29). El procedimiento de escisión electro quirúrgica con asa LEEP es frecuente su 
uso en NIC II y NIC III, se usa con anestesia local y un asa delgada de alambre que se fija a un generador de electrocirugía para extirpar el tejido de interés (30). Cabe destacar que indistintamente el tratamiento que se use, las pacientes deben llevar un seguimiento para observar una correcta respuesta del mismo.

\section{MÉTODO}

$\mathrm{S}$ e realizó una investigación documental de tipo descriptivo, al revisar los programas de DOCcu, con la finalidad de encontrar el cumplimiento por parte de los Sistemas de Salud, donde se registró e interpretó los resultados obtenidos hasta presente fecha. Se utilizaron fuentes bibliográficas certificadas, como repositorios y bases de datos indexadas; dichas fuentes son las siguientes: Pubmed, Scielo, Google Académico. Para la selección de los artículos que cumplieron los criterios inclusión durante el periodo entre enero del 2015 hasta junio 2020 en los idiomas de español e inglés, se utilizaron las palabras clave de los descriptores en ciencias de la salud (DeCs); estos descriptores fueron "Programas Nacionales de Salud", "Evaluación de Programas y proyectos de Salud", "Neoplasias del Cuello Uterino", "Prueba del Papanicolaou". Para el análisis de los artículos originales, primeramente, se procedió la lectura de los resúmenes para evidenciar el cumplimiento de los criterios de inclusión y los de la estrategia PICO (Pacientes, Intervención, Comparación, y Resultados), y tras ello se bajó el documento completo del articulo seleccionado. Los criterios de exclusión fueron información de congresos, simposio, actas, pautas de consensos, artículos incompletos. La búsqueda bibliográfica arrojó 116 investigaciones referentes a los programas de DOCcu; se filtraron 51 artículos por ser duplicados, con textos incompletos, o por no cumplir con los criterios de inclusión. Posteriormente, 65 artículos resultaron elegibles, no obstante, se constató que en función de los criterios de exclusión propuestos en el diseño metodológico no experimental, de la presente investigación, 48 estudios debían ser excluidos; por lo tanto, 17 artículos fueron analizados para cumplir con los objetivos de la investigación, que en su mayoría fueron obtenidos de Pubmed en idioma inglés.

\section{DESARROLLO Y DISCUSIÓN}

$\mathrm{E}$ l MSP dEcuador, implemento la "Estrategia Nacional para el control de cáncer en Ecuador, 2013 - 2023". Los screening de tamizaje de diagnóstico, para la prevención del CCU en los distintos niveles de atención corresponde a Cotest: inmunización, citología convencional y base liquida, citología y test de VPH FDA, triaje de citología en indeterminados + citología seguida de VPH test. FDA. El test de VPH posee una relativa baja especificidad (89\%) para la identificación de lesiones precancerosas. Esta característica del test de VPH hace necesario la aplicación de una segunda prueba (triage) a las mujeres VPH positivo para poder identificar quiénes entre este grupo de mujeres han desarrollado lesiones para que puedan ser diagnosticadas y/o tratadas de manera oportuna (31).

A nivel dEcuador el cumplimiento del programa DOCcu en el 2018 fue del 58,85, quedando aun un 41,15 de mujeres sin la DOCcu; además, el grupo de mujeres en las cuales existió este porcentaje fue el de 45 hasta 49 años de edad. Sin embargo, la cobertura anual del DOCcu., es variante, cuya frecuencia va desde 2011 (17,52\%), 2012 (49,69\%), 2013 (27,91\%), 2014 (19,3\%), 2015 (26,83\%), 2016 (58,45\%), y 
en el 2017 (32,20\%). La cobertura de la "vacuna contra el VPH" comenzó a implementarse desde el año 2014. Se observó un incremento de la inmunización, pero, solo para la edad de 9 años, donde inicio con el $73 \%$ en el 2014, hasta llegar al $84,9 \%$ en el año 2018; en cambio, para la edad de 10 años en el año 2014 fue 70,1\%, y en el 2018 no hay registros; igualmente, para la edad de 11 años en el año 2014 fue $68,9 \%$, seguido en el 2015 con 1,8\%, y en los siguientes años no hay datos.

En el año 2019, realizó una búsqueda bibliográfica, donde identificó 4272 artículos procedentes de Ghana, Malawi, Mozambique, Tanzania, Uganda, Zambia, Zimbabwe y Nigeria (32). Los autores determinaron que la falta de comprensión del riesgo personal o, la educación insuficiente sobre CCU, la falta de conciencia sobre los servicios preventivos, puede llevar a la disminución del cumplimiento del programa DOCcu. Las percepciones erróneas sobre los servicios conocidos también pueden generar temores injustificados, como suposiciones de que los procedimientos utilizados para detectar o tratar el cáncer de cuello uterino son incómodos o que la limpieza del instrumento es deficiente. En relación a las barreras que tiene el servicio del DOCcu, el 89\% señalaron la falta de oferta, el $47 \%$ indicaron la dificultad en el acceso, y la aceptación para la realización del mismo fue solo del $18 \%$.

En el año 2018, en Cuenca, en una población de 4.433 mujeres, reportó una cobertura para la citología convencional del 64,4\% (2.855), y sin cobertura el $35,6 \%$ (33). Los factores relacionados al no acceso al programa DOCcu, fueron la falta de privacidad, el miedo, el maltrato de personal, la falta de tiempo, y la demora en la entrega de resultados.
Se determinó en el año 2015, la calidad del programa DOCcu, en la ciudad de Ambato. La cobertura global en la población fue del 32,5\% (34); en cambio, por grupos de edad de 35 a 64 años fue del $17 \%$, lo que indica, que cuando el riesgo de tener cáncer de cérvix es mayor, 2 de cada 10 mujeres se realizan un test de Papanicolaou. Así mismo, ENSANUT, (35) en el año 2018, reportó que en Ecuador el $54,8 \%$ de mujeres de 12 a 49 años se han realizado alguna vez un examen de Papanicolaou; igualmente se observa que no cubre el total de los grupos de edad en los cuales se les recomienda este examen que es desde los 21 hasta 65 años. Sin embargo, si comparamos con el año 2014 esta cobertura fue del 19,3\% de primeras consultas para DOCcu, realizadas por un médico en los establecimientos de salud, según regiones y provincia; en el año 2013 fue de 27,9\%, en el 2012 que reportó 49,69\%; en el 2011, la cobertura nacional fue del 17, 52\% $(35,36)$.

En el año 2012 en su estudio realizado en el instituto de cáncer de SOLCA Cuenca, validó la sensibilidad y la especificidad de la citología en base líquida comparada con la citología convencional (37), los resultados encontrados fueron una sensibilidad del $62,50 \%$ y una especificidad del 98,04\%. Ademas, encontraron que los genotipos AR mas frecuentes fueron el 56 y 39 (3).

En el año 2017 en Quito, determinaron que la edad de la vacunación depende más del inicio de las relaciones sexuales (IVSA) de la población (39); además, se realizó una encuesta a un grupo de 202 participantes, que se encontraban en la edad de 15 hasta los 25 años, se evidenció que el $60 \%$ (n 121) no se ha vacunado contra el VPH; finalmente concluye que la falta de promoción y la gratuidad de los 
servicios favorecen al aumento de los casos del CCU.

En el año 2019, en Quito, realizó un estudio en 100 gestantes usuarias del hospital Isidro Ayora, para determinar la prevalencia del VPH AR. (40), en mujeres de 20 a 35 años de edad. Las pruebas PCR estimó la presencia de 49 (28\%) pacientes positivas para el VPH AR, los genotipos mas frecuentes fueron el 16 (50\%), 52 (29\%), y 58 (14\%).

En el año 2019 evaluó en la relación costo-efectividad de los programas de DOCcu en las mujeres con VPH positivo del El Salvador (41), en relación con la detección basada en Papanicolaou. La población fueron 8000 participantes, divididas en 2 grupos de edad. Mujeres de 30 y 65 con HPV positivo se les hizo crioterapia y control de cada 5 años, y otro grupo de mujeres de 20 a 65 años quienes tuvieron control cada 2 años, las que resultaron positivo al Paptest se les indicó colposcopia. Los resultados fueron: el riesgo de cáncer se redujo en un 58,5\%, ya que las pruebas de VPH seguidas de crioterapia para mujeres elegibles positivas para el VPH fueron la estrategia menos costosa y más efectiva (US \$ 490 por año de vida salvada) en comparación con el Paptest cada 2 años. Conclusión: el cribado basado en el VPH seguido de crioterapia inmediata en todas las mujeres elegibles sería muy rentable en El Salvador.

Realizó una revision sistematica, donde el 93\% de los artículos fueron de países en desarrollo, 369017 participantes cumplieron los criterios de inclusión: 29 ECA y 4 estudios observacionales (42). Comparó el autoexamen del VPH, con la toma realizada en un consultorio médico, como opciones a los programas del DOCcu. El metanálisis encontró una mayor aceptación entre las participantes del automuestreo del VPH (sobre todo las mujeres de 50 a 60 años), en comparación con el realizado en la consulta médica (que prefirieron las mujeres de 30 a 40 años) (RR: 2,13; IC del 95\%: 1,89 a 2,40). En conclusión, el auto-muestreo del VPH tiene el potencial de aumentar la captación de pruebas de detección de cáncer cervical. En el metanálisis, los kits de automuestreo del VPH enviados directamente a los hogares de mujeres u ofrecidos por un trabajador de la salud resultaron en una mayor aceptación de la detección, en comparación con el estándar de atención.

En el año 2017 en EEUU, reportó que una mejor asociación e integración de las intervenciones en el sistema de salud pública y atención primaria, puede cambiar el contexto dentro del cual ocurren las enfermedades crónicas y reducir las disparidades de salud (43). Las intervenciones del trabajador de salud comunitaria y navegador de pacientes implementadas en centros de salud calificados por el MSP, son efectivas para aumentar el cribado del cáncer y la puntualidad de la resolución del diagnóstico entre las poblaciones con servicios médicos insuficientes. Reducir la brecha entre la comunidad y la clínica es fundamental para apoyar a las comunidades desfavorecidas para obtener acceso a la atención primaria, incluida la detección del cáncer.

En el año 2019 en Ruanda se aplicó una encuesta a 96 médicos generales que trabajaban en hospitales de cuidados paliativos en pacientes con cáncer, de los cuales solo 73 respondieron (76\%) (44). La mayoría de los encuestados tenían entre 25 y 29 años ( $\mathrm{n}=64$ [88\%]) y hombres $(\mathrm{n}=56$ [79\%]), la mayoría llevaba laborando entre 6 a 12 meses. Se identificaron brechas significativas en el conocimiento del cáncer y las habilidades. 
Los médicos identificaron además múltiples causas de retrasos a nivel del sistema. Cuarenta y siete (49\%) médicos identificaron la falta de servicios de patología o detección, 52 de los encuestados informaron que carecen de servicios de patología en sus instalaciones, y solo el 5,8\% de los médicos generales informaron la disponibilidad regular de biopsia. En términos de educación e instrucción de pregrado en habilidades de diagnóstico físico, más de la mitad de todos los encuestados ( $\mathrm{n}=53$ [72,6\%]) deseaban más instrucción durante la escuela de medicina sobre detección de lesiones cervicales y realización de exámenes pélvicos, $34 \quad(46,6 \%)$ deseaban más instrucción para realizar exámenes clínicos de los senos y 31 (42,5\%). (45).

\section{Discusión}

En Ecuador existe el programa de detección oportuna del cáncer de cuello uterino, en él se utiliza la citología convencional que es la obtención de la muestra por la técnica de Papanicolaou; examen que se realiza a todas mujeres a partir del año del inicio de su vida sexual. Sin embargo, la cobertura del programa en el año 2018 a nivel nacional fue del $58,80 \%$. Datos que se comparan por regiones como la ciudad de Cuenca, donde la cobertura fue del $64,4 \%$, en Ambato del $32,5 \%$ y, en esa misma ciudad, para la edad de 35 a 64 años, donde son más frecuentes las lesiones intraepitelial del cuello uterino, la cobertura fue del $17 \%$. Asimismo, ENSANUT informó en ese mismo año que el $54,8 \%$ de las mujeres ecuatorianas se ha realizado un Papanicolaou en algún momento de su vida. Esta baja cobertura puede deberse a la falta de promoción de los programas de DOCcu, a la demora de la entrega de los resultados, además de la falta de los especialistas encargados para el análisis cito-patológico en las unidades de salud.

\section{CONCLUSIÓN}

$\mathrm{E}$ l nivel de cobertura de los programas de DOCcu dependen de la confianza que tengan a las usuarias a los centros de salud, la educación y la socialización, nivel de conocimientos, y la relación entre la usuaria con el personal de salud. Sin embargo, en Ecuador, la cobertura del programa DOCcu., es variable e insuficiente; al contrario, existe una buena aceptación para la "vacuna contra el VPH para las niñas de 9 años", pero para las otras edades esta cobertura es baja nula. En Ecuador, existe un subregistro de la información, falta promoción y además el considerar otras acciones para que las usuarias accedan a los servicios de salud preventivos como lo hacen países desarrollados, así mismo, en lugares donde no se cuentan con recursos necesarios se pueden implementar por ejemplo la IVAA como lo hizo El Salvador donde los costos por estos programas se han disminuido por la implementación de estas nuevas técnicas.

\section{REFERENCIAS BIBLIOGRÁFICAS}

1. American Cancer Society. Cancer Facts \& Figures. Journal American Cancer Society. 2016 marzo

2. OMS. Papilomavirus humanos (PVH) y cáncer cérvicouterino. Organización Mundial de la Salud; 2019

3. Organización Panamericana de la Salud. Programa nacional de salud reproductiva componente cérvicouterino. Salud. Organización Panamericana de la Salud, Ministerio de salud y asistencia social; 2017

4. American Cancer Society. American Cancer Society. [Online]. 2019 [cited 2020 abril 6. Available from: 
https://www.cancer.org/cancer/cervi cal-cancer/about/key-statistics.html

5. Mendoza. Programa de detección del cáncer cervicouterino: políticas públicas y experiencias de los actores que implementan el programa en el estado de Veracruz, México. Salud Colectiva. 2017 marzo; 13(3)

6. Vargas V, Vargas Aguilar, Tovar JM. Detección primaria del cáncer cérvicouterino. Elsiever. 2015 septiembre; 83(5)

7. Organización Mundial de la Salud. Prevención y control integrales del cáncer cérvicouterino: un futuro más saludable para niñas y mujeres. Whasington DC: OMS; 2013.

8. Subsecretaría de Prevención y Promoción a la Salud. Programa de acción específico 2007-2012 cáncer cérvicouterino. México: Subsecretaría de Prevención y Promoción a la Salud., Secretaría de Salud; 2008

9. SOLCA. Cáncer de cuello uterino. SOLCA. 2017 noviembre

10. Ministerio de Salud Pública. Estrategia nacional para la atención integral del cáncer en Ecuador. Quito: Dirección Nacional de Estrategias de Prevención y Control, Subsecretaria Nacional de Vigilancia de la Salud Pública; 2017

11. OMS, CDC. Caja de herramientas para los programas de prevención y control del cáncer cérvicouterino. Luxemburgo: Organización Mundial de la Salud, Centros para el Control y la Prevención de Enfermedades; 2017

12. Orquera A. et al. Calidad del Programa de Detección Oportuna del Cáncer Cérvicouterino. Enfermería Investiga, Investigación, Vinculación, Docencia y Gestión. 2016 octubre; 1(4)

13. Gúzman $N$, Rojas $M$. Factores que influyen en la cobertura del programa de detección oportuna del cáncer cérvicouterino en mujeres de edad fértil que acuden al Subcentro de Salud El Valle, Cuenca 2017. Universidad Católica de Cuenca. 2018 abril

14. OPS. Prevención y control integrales del cáncer cérvicouterino: un futuro más saludable para niñas y mujeres.
Organización Panamericana de la

Salud; 2016

15. Cabrera V. JA, Cárdena H. OJ, Campoverde C. MA, Ortíz S. JI. Prevalencia de genotipos del papiloma virus humano en mujeres de la provincia del Azuay, Ecuador. MASKANA. 2015 Jun 25;6(1):79-93

16. Ferlay J, Shin H, Bray F, Forman D, Mathers C, Parkin D. Cancer Incidence and Mortality Worldwide. International Agency for Research on Cancer, GLOBOCAN; 2015

17. Planificador de Acción de Prevención del Cáncer. Planificador de Acción para la Prevención del Cáncer del Cáncer de Cuello. [Online]; 2015 [cited 2018 abril 15. Available from: rho.org/aps/learnbasics.htm

18. Kumar M, Path F, Abbas, Fausto, Aster. Cervix premalignant and malignant neoplasias. 8th ed. Disease R\&CPBo, editor.: Elsiever; 2013

19. Sánchez J. Mortalidad por cáncer cervicouterino. Gaceta Médica de México. 2012 febrero; 148

20. Sánchez J. Mortalidad por cáncer cervicouterino. Gaceta Médica de México. 2012 febrero; 148

21. Moscicki A, Schiffman M, Kjaer S, Villa L. Updating the natural history of VPH and anogenital cancer. Vaccine. 2016 septiembre; 3(3).

22. Solomon D, David D, Kurman R. The Bethesda System: terminology for reporting result of cervical cytology. Jama. 2011 marzo; 287

23. IARC. IARC Handbooks of Cancer Prevention. 10th ed. Press L, editor: Cervix Cancer Screening; 2010

24. OMS. WHO guidelines for screening and treatment of precancerous lesions for cervical cancer prevention. Salud. World Health Organization; 2013. Report No.: ISBN 978-92-75-31833-1

25. OMS. National Cancer Control Programmes: Policies and managerial guidelines. Salud. Genova: World Health Organization; 2002

26. Ferlay J, Ervik M, Lam F, Colombet M, Mery L, Piñeros M, et al. Observatorio 
Global del Cáncer: Cáncer Hoy. Francia: Agencia Internacional para la Investigación del Cáncer

27. American Cancer Society. Cancer Facts and Figures. Atlanta: American Cáncer Society; 2019

28. Sánchez. Tamizaje y tratamiento de las lesiones precancerosas para la prevención del cáncer cérvicouterino. Revista Médica Sinergia. 2019 noviembre; 4(11)

29. Concepción J, Álvarez E, Reyes 0. Tratamiento observacional vs. Ablativo de las lesiones intraepiteliales escamosas de bajo grado. Estudio de cohorte pareado retrospectivo. Clínica e Investigación en Ginecología y Obstetricia. 2017 junio

30. Orosco L, Tristan M, Beita A, Vreugdenhil M, Andrino R, Aguero C, et al. Guia de práctica clínica para el manejo de lesiones premalignas del cuello uterino. 1ra Edición. Salvat Editores, editor. Vol. 1. Costa Rica; 45$66 \mathrm{p}$

31. Martin-Hirsch P, Paraskevaidis E, Bryant A, Dickison H. Surgery for cervical intraepithelial neoplasia. Cochrane Database of Systematic Reviews. 2013 diciembre

32. Ministerio de Salud Publica G de E. Estrategia Nacional para la atención del cáncer en Ecuador [Internet]. 2017 [cited 2020 Jun 13]. p. 53. Available from:

https://aplicaciones.msp.gob.ec/salud/ archivosdigitales/documentosDireccio nes/dnn/archivos/ac_0059_2017.pdf

33. Rahman R, Clark MD, Collins Z, Traore $\mathrm{F}$, Dioukhane EM, Thiam $\mathrm{H}$, et al. Cervical cancer screening decentralized policy adaptation: an African ruralcontext-specific systematic literature review. Vol. 12, Global Health Action. Taylor and Francis Ltd.; 2019

34. Guzmán N, Rojas M. Factores que influyen en la cobertura del programa de detección oportuna del cáncer cérvicouterino en mujeres de edad fértil que acuden al Subcentro de Salud
El Valle [Tesis de grado]. Universidad de Cuenca; 2018

35. Andrade EAO, Cepeda ML. Calidad del Programa de Detección Oportuna del Cáncer Cérvicouterino, Centro de Salud Cevallos, Junio 2015 a Junio 2016. Enfermería Investig. 2016 Dec 18;1(4 Dic):158-63

36. Instituto Nacional de Estadisticas y Censos GE. Encuesta Nacional de Salud y Nutrición ENSANUT. Quito; 2018

37. Vega $B$, Sacoto catalina. Prevalencia de cáncer de cuello uterino en Ecuador y estrategias para su reducción. Rev la Fac Ciencias Médicas la Univ Cuenca. 2012;46-51.

38. Escobar Landivar C estefanía, Jerves Carrasco RE. Citología Convencional vs Monocapa para LIEBG-LIEAG en el Instituto del Cáncer SOLCA - Cuenca. Universidad del Azuay; 2012.

39. Ministerio de Salud Pública G del E. Esquema Nacional de Vacunacion Ecuador 2019. Quito; 2019

40. Acosta M, Merino N, Endara M, Padilla M, Valdivieso Vélez L. Virus del Papiloma Humano-Tres vacunas por una vida Campaña de concientización y prevención del VPH. Quito: Quito: USFQ, 2017; 2017

41. Morales G, Carlos Cazar J. Participación de la Universidad Central dEcuador en la atención médica y humanitaria duran- te la crisis social de octubre 2019. Rev la Fac Ciencias Médicas. 2019 Dec 2;44(1):86-8

42. Campos NG, Maza M, Alfaro K, Gage JC, Castle PE, Felix JC, et al. The costeffectiveness of implementing HPV testing for cervical cancer screening in El Salvador. Int J Gynecol Obstet. 2019 Apr 1;145(1):40-6

43. Yeh PT, Kennedy CE, De Vuyst $H$, Narasimhan M. Self-sampling for human papillomavirus (HPV) testing: A systematic review and meta-Analysis. Vol. 4, BMJ Global Health. BMJ Publishing Group; 2019 
44. Roland KB, Milliken EL, Rohan EA, Degroff A, White S, Melillo S, et al. Use of Community Health Workers and Patient Navigators to Improve Cancer Outcomes among Patients Served by Federally Qualified Health Centers: A Systematic Literature Review. Vol. 1,Health Equity. Mary Ann Liebert Inc.; 2017. p. $61-76$
45. Martin AN, Kaneza K-M, Kulkarni A, Mugenzi P, Ghebre R, Ntirushwa D, et al. Càncer Control at the District Hospital Level in Sub-Saharan Africa: An Educational and Resource Needs Assessment of General Practitioners. J Glob Oncol. 2019 Nov;5(5):1-8 